\title{
ASYMPTOTICS OF A FREE-BOUNDARY PROBLEM
}

\section{F. V. Atkinson, H. G. Kaper, and Man Kam Kwong}

\begin{abstract}
As was shown by [1], there exists a unique $R>0$, such that the differential equation

$$
u^{\prime \prime}+\frac{2 \nu+1}{r} u^{\prime}+u-u^{q}=0, \quad r>0,
$$

$(0 \leq q<1, \nu \geq 0)$ admits a classical solution $u$, which is positive and monotone on $(0, R)$ and which satisfies the boundary conditions

$$
u^{\prime}(0)=0, \quad u(R)=u^{\prime}(R)=0 .
$$

In this article, it is shown that $u(0)$ is bounded, but $R$ grows beyond all bounds as $q \rightarrow 1$.
\end{abstract}

\section{The Problem}

In [2], the reaction-diffusion equation $\Delta u+u^{1 / 2}-1=0$ was proposed as a simple model for Tokamak equilibria with magnetic islands. The equation motivated a study of free-boundary problems for reaction-diffusion equations in $\mathbf{R}^{N}(N=2,3, \ldots)$ of the general form $\Delta u+u^{p}-u^{q}=0$, where $0 \leq q<p \leq 1$. In [1], we showed that there is a unique $R(R>0)$ and a unique positive-valued function $u$ on $(0, R)$ such that $u$ is the radial solution of the differential equation which satisfies the boundary conditions $u(R)=0, u^{\prime}(R)=0$. (A radial solution depends only on the radial variable $r=|x|$.) The solution $(R, u)$ of the free-boundary problem depends on the values of the exponents $p$ and $q$.

In this article, we analyze the special case $p=1$ in more detail and focus on the behavior of the solution as $q \rightarrow 1$. That is, we are interested in the behavior as $q \rightarrow 1$ $(q<1)$ of the pair $(R, u), R$ a real number $(R>0), u$ a positive-valued function on $(0, R)$, which satisfies the boundary-value problem

$$
\begin{gathered}
u^{\prime \prime}+\frac{2 \nu+1}{r} u^{\prime}+u-u^{q}=0, \quad 0<r<R, \\
u^{\prime}(0)=0, \quad u(R)=u^{\prime}(R)=0 .
\end{gathered}
$$

We consider $\nu$ as a real number, not necessarily half-integer $(\nu \geq 0)$. The existence and uniqueness of such a solution follow from [1]. The function $u$ is monotone on $(0, R)$.

\section{The Result}

We prove the following result.

Theorem 1. For each $q \in[0,1)$, there is a unique $R>0$ such that (1.1), (1.2) admits a (classical) solution $u$ that is positive everywhere on $(0, R)$. The function $u$

Received January 12, 1995, revised June 26, 1995.

1991 Mathematics Subject Classification. Primary 34B15. Secondary 35J25, 35J65.

Key words and phrases: reaction-diffusion equation, free-boundary problem, radial solution, Emden-Fowler equation, positive solution, asymptotic behavior. 
is monotonically decreasing on $(0, R) ; u(0)$ is bounded, but $R$ grows beyond bounds as $q \rightarrow 1$.

In the special case $\nu=\frac{1}{2}(N=3)$, we have a lower bound on $R$,

$$
R>\sqrt{\frac{2}{1-q}}, \quad 0 \leq q<1 .
$$

However, as we do not have a comparable upper bound, we cannot conclude that $R=\mathrm{O}\left((1-q)^{-1 / 2}\right)$ as $q \rightarrow 1$.

\section{The Proof}

Using a shooting argument, we replace the boundary-value problem (1.1), (1.2) by the initial-value problem

$$
\begin{gathered}
u^{\prime \prime}+\frac{2 \nu+1}{r} u^{\prime}+u-u^{q}=0, \quad r>0, \\
u(0)=\gamma, \quad u^{\prime}(0)=0 .
\end{gathered}
$$

The results of [1] imply that, for any $q \in[0,1)$, there is a unique $\gamma>1$ such that the solution of (3.1), (3.2) decreases from $\gamma$ to meet the $r$-axis with zero slope at some value $R>0$. Denoting this solution by $u(\cdot, \gamma)$, we have

$$
u(R, \gamma)=0, \quad u^{\prime}(R, \gamma)=0
$$

The lower bound on $\gamma$ can be sharpened to $(2 /(1+q))^{1 /(1-q)}$, but 1 suffices for our purpose. The proof consists of a detailed investigation of the behavior of $u(\cdot, \gamma)$.

3.1. Down to 1 . We begin by showing that $u(r, \gamma)$ decreases monotonically from the value $\gamma$ at $r=0$ to the value 1 at some finite point $r_{0}$.

Lemma 1. There exists a point $r_{0}<j_{\nu, 1} /(1-q)^{1 / 2}$ such that $u(\cdot, \gamma)$ is monotonically decreasing on $\left(0, r_{0}\right)$ with $u\left(r_{0}, \gamma\right)=1$ and $u^{\prime}\left(r_{0}, \gamma\right)<0$. Here, $j_{\nu, 1}$ is the first positive zero of $J_{\nu}$-the Bessel function of the first kind of order $\nu$.

Proof. As long as $u>1$, we have $u-u^{q}>(1-q) u$, so $u(\cdot, \gamma)$ oscillates faster than the solution $v$ of the equation

$$
v^{\prime \prime}+\frac{2 \nu+1}{r} v^{\prime}+(1-q) v=0 .
$$

In particular, $u(\cdot, \gamma)$ reaches the value 1 before $v$ does. Now, $v(r)$ is a constant multiple of $r^{-\nu} J_{\nu}\left(r(1-q)^{1 / 2}\right)$ where $J_{\nu}$ is the Bessel function of the first kind of order $\nu$-see, for example, [3]. Hence, $v(r)=1$ for some value $r<j_{\nu, 1} /(1-q)^{1 / 2}$ where $j_{\nu, 1}$ is the first positive zero of $J_{\nu}$. We conclude that there must be a point $r_{0}<j_{\nu, 1} /(1-q)^{1 / 2}$ such that $\gamma>u(r, \gamma)>1$ for $0<r<r_{0}$ and $u\left(r_{0}, \gamma\right)=1$.

Since $u^{\prime}(0, \gamma)=0$ and $u^{\prime \prime}(r, \gamma)<0$ near 0 , it must be the case that $u^{\prime}(r, \gamma)<0$ near 0.

Suppose $u(\cdot, \gamma)$ were not monotone on $\left(0, r_{0}\right)$. Then there exists a value $r_{1} \in\left(0, r_{0}\right)$ where $u(r, \gamma)$ has a local minimum with $u\left(r_{1}, \gamma\right)>1$. Because $u(r, \gamma)$ reaches the value 1 at $r_{0}$, there then must exist a value $r_{2} \in\left(r_{1}, r_{0}\right)$ such that $u\left(r_{2}, \gamma\right)=u\left(r_{1}, \gamma\right)$ and $u^{\prime}\left(r_{2}, \gamma\right) \leq 0$. Multiplying the differential equation (3.1) by $u^{\prime}$ and integrating over $\left(r_{1}, r_{2}\right)$, we find that

$$
\frac{1}{2}\left(u^{\prime}\left(r_{2}, \gamma\right)\right)^{2}=-(2 \nu+1) \int_{r_{1}}^{r_{2}} \frac{\left(u^{\prime}(r, \gamma)\right)^{2}}{r} d r .
$$


But here we have a contradiction, as the two sides of this identity have opposite signs. Therefore, it must be the case that $u(\cdot, \gamma)$ is monotone on $\left(0, r_{0}\right)$.

The monotonicity of $u(\cdot, \gamma)$ on $\left(0, r_{0}\right)$ implies that $u^{\prime}\left(r_{0}, \gamma\right) \leq 0$. If $u^{\prime}\left(r_{0}, \gamma\right)=0$, then it follows from the Lipschitz continuity of the function $u-u^{q}$ for $u>0$ and the consequential uniqueness of the solution of the initial-value problem for (3.1) in the direction of decreasing $r$ starting at $r=r_{0}$ that $u(r, \gamma)=1$ for all $r \in\left(0, r_{0}\right)$. But then we have a contradiction, as $u(0, \gamma)=\gamma>1$. We conclude that $u^{\prime}\left(r_{0}, \gamma\right)<0$.

3.2. Beyond $r_{0}$. From Lemma 1 , we know that $u(r, \gamma)$ decreases monotonically until it reaches the value 1 with a negative slope at $r=r_{0}$. Beyond $r_{0}, u(r, \gamma)$ decreases further until either it reaches the value 0 with a negative or zero slope or it bottoms out at some finite value of $r$ with a minimum value between 0 and 1 .

Let $r_{1}$ be the point where $u(r, \gamma)$ ceases to be positive,

$$
r_{1}=\sup \left\{r>r_{0}: u(s, \gamma)>0, \quad 0<s<r\right\} .
$$

If $r_{1}$ is finite and $u\left(r_{1}, \gamma\right)=0$, we do not consider $u(\cdot, \gamma)$ beyond $r_{1}$. In this case, we can use the same argument as in the proof of Lemma 1 to show that $u(\cdot, \gamma)$ is monotonically decreasing on the entire interval $\left(0, r_{1}\right)$. In particular, if $\gamma$ is such that not only $u\left(r_{1}, \gamma\right)=0$, but also $u^{\prime}\left(r_{1}, \gamma\right)=0$, then $u(\cdot, \gamma)$ defines the (unique) solution $u$ of the free-boundary problem (3.1), (3.3), where $R=r_{1}$.

If $r_{1}=\infty$, then $u(r, \gamma)$ has a positive minimum at some finite value of $r$, after which it oscillates with decreasing amplitude around the constant value 1 .

Lemma 2. For $0<r<r_{1}$, we have $0<u(r, \gamma)<\gamma$.

Proof. The lemma is true for $0<r \leq r_{0}$ (cf. Lemma 1). Beyond $r_{0}$, we use a simple energy argument. The energy $E$ of any solution $u$ of (3.1), defined by the expression

$$
E(r)=\frac{1}{2}\left(u^{\prime}(r)\right)^{2}+\frac{1}{2}(u(r))^{2}-\frac{1}{q+1}(u(r))^{q+1},
$$

is a monotonically decreasing function of its argument since

$$
E^{\prime}(r)=-\frac{2 \nu+1}{r}\left(u^{\prime}(r)\right)^{2} \leq 0
$$

for all $r \geq 0$.

Suppose the lemma were false for $r_{0}<r<r_{1}$. Then $u\left(r_{2}, \gamma\right)=\gamma$ for some $r_{2} \in$ $\left(r_{0}, r_{1}\right)$ where $E\left(r_{2}\right) \geq \gamma^{2} / 2-\gamma^{q+1} /(q+1)=E(0)$, and we have a contradiction.

Let $w$ be defined in terms of $u(\cdot, \gamma)$ by the expression

$$
w(r)=\frac{r u(r, \gamma)}{\gamma}
$$

This function is a solution of the initial-value problem

$$
\begin{gathered}
w^{\prime \prime}+\frac{2 \nu-1}{r} w^{\prime}+\left(1-\frac{1}{(u(r, \gamma))^{1-q}}-\frac{2 \nu-1}{r^{2}}\right) w=0, \quad r>0 \\
w(0)=0, \quad w^{\prime}(0)=1 .
\end{gathered}
$$

It vanishes when $u$ vanishes, while its derivative vanishes when both $u$ and $u^{\prime}$ vanish. Furthermore,

$$
0<w(r)<r, \quad 0<r<r_{1} .
$$

The following lemma gives a lower bound for $r_{1}$. 
Lemma 3. We have $r_{1}>j_{\nu, 1} / \delta$ where

$$
\delta=\sqrt{1-\gamma^{-(1-q)}} .
$$

Proof. Because $u(r, \gamma)<\gamma, w$ oscillates less than the solution $v$ of the initial-value problem

$$
v^{\prime \prime}+\frac{2 \nu-1}{r} w^{\prime}+\left(\delta^{2}-\frac{2 \nu-1}{r^{2}}\right) v=0, \quad r>0 ; \quad v(0)=0, \quad v^{\prime}(0)=1,
$$

at least as long as $v$ is positive. Since $v(r)=2^{\nu} \Gamma(\nu+1) \delta^{-\nu} r^{1-\nu} J_{\nu}(\delta r)$, the first zero of $v$ occurs at $j_{\nu, 1} / \delta$. Therefore, it must be the case that $r_{1}>j_{\nu, 1} / \delta$.

3.3. Bounds on $\left(0, j_{\nu, 1} / \delta\right)$. We rewrite $(3.9)$ in the form

$$
w^{\prime \prime}+\frac{2 \nu-1}{r} w^{\prime}+\left(\delta^{2}-\frac{2 \nu-1}{r^{2}}\right) w=f(w)
$$

where

$$
f(w)=\frac{1}{\gamma^{1-q}}\left\{\left(\frac{r}{w}\right)^{1-q}-1\right\} w .
$$

Using the method of variation of parameters, we obtain the integral equation for $w$,

$$
w(r)=r g(\delta r)+\frac{\pi}{2} \int_{0}^{r} r^{1-\nu} s^{\nu}\left\{J_{\nu}(\delta s) Y_{\nu}(\delta r)-Y_{\nu}(\delta s) J_{\nu}(\delta r)\right\} f(w(s)) d s
$$

where

$$
g(\rho)=2^{\nu} \Gamma(\nu+1) \rho^{-\nu} J_{\nu}(\rho) .
$$

The notation $J_{\nu}$ and $Y_{\nu}$ are the Bessel functions of the first and second kind, respectively, of order $\nu$. The expression (3.16) holds for all $r \in\left[0, r_{1}\right)$ or, if $r_{1}$ is finite, for all $r \in\left[0, r_{1}\right]$. We now restrict $r$ to the interval $\left[0, j_{\nu, 1} / \delta\right]$.

Lemma 4. For $0<r<j_{\nu, 1} / \delta$, we have

$$
0<r g(\delta r)<w(r)<r\left(g(\delta r)+\frac{\phi(\delta r)}{\log \gamma}\right)
$$

where $g$ is defined in (3.17) and

$$
\phi(\rho)=\frac{\rho^{2}(g(\rho))^{-1} \log (g(\rho))^{-1}}{4(\nu+1)} .
$$

Proof. Take any $r \in\left(0, j_{\nu, 1} / \delta\right)$. It follows from the Kneser-Sommerfeld expansion [3, Section 15.42] that

$$
J_{\nu}(\delta s) Y_{\nu}(\delta r)-Y_{\nu}(\delta s) J_{\nu}(\delta r)=\frac{4 \delta r J_{\nu}(\delta r)}{\pi J_{\nu}(\delta s)} \sum_{n=1}^{\infty} \frac{\left(J_{\nu}\left(j_{\nu, n} s / r\right)\right)^{2}}{\left(j_{\nu, n}^{2}-(\delta r)^{2}\right) j_{\nu, n}{J_{\nu}^{\prime}}^{2}\left(j_{\nu, n}\right)}
$$

for $0 \leq s \leq r$. All the terms on the right-hand side are positive, so the expression on the left-hand side is positive. Furthermore, $f(w(s))$ is positive for $0 \leq s \leq r$. Therefore, the integral in (3.16) is positive. Obviously, $g(\delta r)$ is positive, so

$$
w(r)>r g(\delta r)>0, \quad 0<r<j_{\nu, 1} / \delta .
$$

It remains to establish the upper bound on $w(r)$ in (3.18). From (3.21) and the fact that $g$ is decreasing on $\left(0, j_{\nu, 1}\right)$, we deduce that

$$
\frac{s}{w(s)}<\frac{1}{g(\delta s)} \leq \frac{1}{g(\delta r)}, \quad 0 \leq s \leq r
$$


Therefore,

$$
f(w(s))<\frac{(g(\delta r))^{-(1-q)}-1}{\gamma^{1-q}} w(s), \quad 0 \leq s \leq r .
$$

Furthermore, $w(s) \leq s$, cf. (3.11), so

$$
\begin{aligned}
& w(r)-r g(\delta r)=\frac{\pi}{2} \int_{0}^{r} r^{1-\nu} s^{\nu}\left\{J_{\nu}(\delta s) Y_{\nu}(\delta r)-Y_{\nu}(\delta s) J_{\nu}(\delta r)\right\} f(w(s)) d s \\
& \leq r \frac{(g(\delta r))^{-(1-q)}-1}{\gamma^{1-q}-1}\left[\rho^{-\nu} \frac{\pi}{2} \int_{0}^{\rho}\left\{J_{\nu}(z) Y_{\nu}(\rho)-Y_{\nu}(z) J_{\nu}(\rho)\right\} z^{\nu+1} d z\right]_{\rho=\delta r}
\end{aligned}
$$

The expression in square brackets can be evaluated by means of the recurrence formulae for Bessel functions [3, Section 3.2] and the resulting expression can be.simplified further by means of the Wronskian [3, Section 3.63],

$$
\rho^{-\nu} \frac{\pi}{2} \int_{0}^{\rho}\left\{J_{\nu}(z) Y_{\nu}(\rho)-Y_{\nu}(z) J_{\nu}(\rho)\right\} z^{\nu+1} d z=1-g(\rho) .
$$

We estimate this expression by substituting the series expansion for the Bessel function $J_{\nu}$ and truncating after the first term,

$$
1-g(\rho)=1-2^{\nu} \Gamma(\nu+1) \rho^{-\nu} J_{\nu}(\rho) \leq \frac{\rho^{2}}{4(\nu+1)} .
$$

Thus,

$$
\left[\rho^{-\nu} \frac{\pi}{2} \int_{0}^{\rho}\left\{J_{\nu}(z) Y_{\nu}(\rho)-Y_{\nu}(z) J_{\nu}(\rho)\right\} z^{\nu+1} d z\right]_{\rho=\delta r} \leq \frac{(\delta r)^{2}}{4(\nu+1)}
$$

To estimate the factor in front of the square brackets in (3.24), we observe that $0<$ $g(\delta r)<1$ on $\left(0, j_{\nu, 1} / \delta\right)$ and $\gamma>1$. Furthermore, one readily verifies that

$$
\frac{1-x^{1-q}}{\log x^{-1}} \leq \frac{y^{1-q}-1}{\log y}
$$

for any pair $(x, y)$ with $0<x \leq 1 \leq y$. Therefore,

$$
\begin{aligned}
& \frac{(g(\delta r))^{-(1-q)}-1}{\gamma^{1-q}-1}=(g(\delta r))^{-(1-q)} \frac{1-(g(\delta r))^{1-q}}{\gamma^{1-q}-1} \\
& \quad \leq \frac{(g(\delta r))^{-(1-q)} \log (g(\delta r))^{-1}}{\log \gamma} \leq \frac{(g(\delta r))^{-1} \log (g(\delta r))^{-1}}{\log \gamma}
\end{aligned}
$$

Using (3.27) and (3.28) in (3.24), we obtain the estimate

$$
w(r)-r g(\delta r) \leq r \frac{\phi(\delta r)}{\log \gamma}
$$

where $\phi$ is defined in (3.19). The upper bound for $w(r)$ given in (3.18) follows.

In terms of $u$, we have the bounds

$$
0<\gamma g(\delta r)<u(r, \gamma)<\gamma\left[g(\delta r)+\frac{\phi(\delta r)}{\log \gamma}\right], \quad 0<r<\frac{j_{\nu, 1}}{\delta} .
$$

Because $\phi(\rho)$ increases beyond bounds as $g(\rho)$ decreases to 0 , the upper bound in (3.18) or (3.30) increases indefinitely as $r$ approaches the right endpoint of the interval $\left(0, j_{\nu, 1} / \delta\right)$ 
In the following analysis, we also need an estimate of the quantity $r^{1-2 \nu}\left(r^{2 \nu-1} w\right)^{\prime}$ $(r)$. It is given by the expression

$$
\begin{aligned}
& r^{1-2 \nu}\left(r^{2 \nu-1} w\right)^{\prime}(r)= \\
& \quad h(\delta r)+\delta \frac{\pi}{2} \int_{0}^{r} r^{1-\nu} s^{\nu}\left\{J_{\nu}(\delta s) Y_{\nu-1}(\delta r)-Y_{\nu}(\delta s) J_{\nu-1}(\delta r)\right\} f(w(s)) d s
\end{aligned}
$$

where

$$
h(\rho)=2^{\nu} \Gamma(\nu+1) \rho^{1-\nu} J_{\nu-1}(\rho) .
$$

Like (3.16), (3.31) holds for all $r \in\left[0, r_{1}\right)$ or, if $r_{1}$ is finite, for all $r \in\left[0, r_{1}\right]$. The following lemma gives an estimate on $\left(0, j_{\nu, 1} / \delta\right)$.

Lemma 5. For $0<r<j_{\nu, 1} / \delta$, we have

$$
\left|r^{1-2 \nu}\left(r^{2 \nu-1} w\right)^{\prime}(r)-h(\delta r)\right|<2(\nu+1) \frac{\phi(\delta r)}{\log \gamma}
$$

where $h$ is defined in (3.32) and $\phi$ is defined in (3.19).

Proof. The proof is similar to, although slightly more involved than, the proof of Lemma 4. Instead of (3.16), we use (3.31). The analog of (3.24) is

$$
\begin{aligned}
& \delta \frac{\pi}{2} \int_{0}^{r} r^{1-\nu} s^{\nu}\left\{J_{\nu}(\delta s) Y_{\nu-1}(\delta r)-Y_{\nu}(\delta s) J_{\nu-1}(\delta r)\right\} f(w(s)) d s \\
& \quad \leq \frac{(g(\delta r))^{-(1-q)}-1}{\gamma^{1-q}-1}\left[\rho^{1-\nu} \frac{\pi}{2} \int_{0}^{\rho}\left\{J_{\nu}(z) Y_{\nu-1}(\rho)-Y_{\nu}(z) J_{\nu-1}(\rho)\right\} z^{\nu+1} d z\right]_{\rho=\delta r} .
\end{aligned}
$$

The expression in square brackets again can be evaluated; instead of (3.25) we have

$$
\rho^{1-\nu} \frac{\pi}{2} \int_{0}^{\rho}\left\{J_{\nu}(z) Y_{\nu-1}(\rho)-Y_{\nu}(z) J_{\nu-1}(\rho)\right\} z^{\nu+1} d z=2(\nu+1)-h(\rho)
$$

where

$$
2(\nu+1)-h(\rho)=2(\nu+1)-2^{\nu} \Gamma(\nu+1) \rho^{1-\nu} J_{\nu-1}(\rho) \leq \frac{1}{2} \rho^{2} .
$$

The lemma follows from (3.31), (3.34), (3.35), (3.36), and (3.27).

3.4. Estimates at $r_{0}$. We use the results of Lemmas 4 and 5 to estimate $r_{0}$ and $r^{1-2 \nu}\left(r^{2 \nu-1} w\right)^{\prime}$ at $r_{0}$.

Lemma 6. Let $a \in\left(j_{\nu-1,1}, j_{\nu, 1}\right)$ be fixed. Then, there exists a constant $\gamma_{1}>1$ that does not depend on $q$ such that

$$
r_{0}^{1-2 \nu}\left(r^{2 \nu-1} w\right)^{\prime}\left(r_{0}\right)<-\frac{1}{2}|h(a)|
$$

and

$$
\frac{a}{\delta}<r_{0}<\left(1+\frac{4 \nu}{|h(a)|}\right)^{1 /(2 \nu)} \frac{a}{\delta}
$$

for all $\gamma \geq \gamma_{1}$.

Proof. With the choice of $a$ indicated in the statement of the lemma, we have $g(a)>0$ and $h(a)<0$. These inequalities follow from the interlacing property of the zeros of Bessel functions,

$$
0<j_{\nu, 1}<j_{\nu+1,1}<j_{\nu, 2}<j_{\nu+1,2}<j_{\nu, 3}<\cdots,
$$

cf. [3, Section 15.22]. 
We begin by observing that $w$ oscillates less than $v$ where $v(r)=r g(\delta r)$ with $g$ defined by (3.17). Therefore, $r_{0}$, which is defined by the identity $w(r)=r / \gamma$, is certainly beyond the point $r_{2}$ where $g\left(\delta r_{2}\right)=1 / \gamma$. Therefore, if

$$
\gamma_{0}=1 / g(a)
$$

then $g\left(\delta r_{2}\right) \leq g(a)$ for all $\gamma \geq \gamma_{0}$. Now, $g$ is monotonically decreasing between $a$ and $j_{\nu-1,1}$, so we also have $\delta r_{2} \geq a$ for all $\gamma \geq \gamma_{0}$. Since $r_{0}>r_{2}$, we thus have shown that

$$
a / \delta<r_{0} \text {. }
$$

for all $\gamma \geq \gamma_{0}$.

With $r_{3}=a / \delta$, it follows from (3.33) that

$$
r_{3}^{1-2 \nu}\left(r^{2 \nu-1} w\right)^{\prime}\left(r_{3}\right)<-|h(a)|+2(\nu+1) \frac{\phi(a)}{\log \gamma} .
$$

Here, $h(a)$ and $\phi(a)$ do not depend on $q$ or $\gamma$. Therefore, if we now define $\gamma_{1}$ by

$$
\gamma_{1}=\min \left\{\gamma_{0}, \exp \left(2(\nu+1) \frac{\phi(a)}{|h(a)|}\right)\right\}
$$

then $\gamma_{1}$ is independent of $q$ and

$$
r_{3}^{1-2 \nu}\left(r^{2 \nu-1} w\right)^{\prime}\left(r_{3}\right)<-\frac{1}{2}|h(a)|
$$

for all $\gamma \geq \gamma_{1}$. Writing the differential equation (3.9) in the form

$$
\left(r^{1-2 \nu}\left(r^{2 \nu-1} w\right)^{\prime}\right)^{\prime}=-\left(1-u^{-(1-q)}\right)
$$

we observe that the function $r^{1-2 \nu}\left(r^{2 \nu-1} w\right)^{\prime}$ is decreasing as long as $u(r, \gamma)>1$ - that is, up to $r_{0}$. Therefore, the bound (3.43) extends to the entire interval $\left[r_{3}, r_{0}\right]$ and we have

$$
r^{1-2 \nu}\left(r^{2 \nu-1} w\right)^{\prime}(r)<-\frac{1}{2}|h(a)|, \quad r_{3} \leq r \leq r_{0}
$$

for all $\gamma \geq \gamma_{1}$. In particular, the inequality holds at $r_{0}$, as asserted in (3.37).

Multiplying both sides of the inequality (3.45) by $r^{2 \nu-1}$ and integrating over the interval $\left(r_{3}, r_{0}\right)$, we find

$$
\left(\frac{w\left(r_{3}\right)}{r_{3}}+\frac{|h(a)|}{4 \nu}\right) r_{3}^{2 \nu}-\frac{|h(a)|}{4 \nu} r_{0}^{2 \nu}>\frac{w\left(r_{0}\right)}{r_{0}} r_{0}^{2 \nu} .
$$

Here, we estimate the expression on the right-hand side from below by 0 . On the left-hand side, we estimate the ratio $w\left(r_{3}\right) / r_{3}$ from above by 1 ; cf. (3.11). Thus,

$$
r_{0}^{2 \nu}<\left(1+\frac{4 \nu}{|h(a)|}\right) r_{3}^{2 \nu}
$$

The inequalities (3.38) now follow from (3.40) and (3.47).

3.5. Down to 0. We are now in a position to prove that the continuation of $u$ beyond $r_{0}$ decreases to 0 for all sufficiently large $\gamma$, independently of $q$.

Lemma 7. There exists a constant $\gamma_{2}$ that does not depend on $q\left(\gamma_{2} \geq \gamma_{1}\right.$, where $\gamma_{1}$ is the constant introduced in Lemma 6), such that $r_{1}<\infty$ for all $\gamma \geq \gamma_{2}$.

Proof. The proof is by contradiction where we assume that, for some $\gamma \geq \gamma_{1}$, the solution $u(\cdot, \gamma)$ of $(3.1),(3.2)$ is positive for all $r \geq 0$. 
Consider the function $w$ defined by (3.8). By assumption, $w$ is positive for all $r>0$. Because $\left(r^{1-2 \nu}\left(r^{2 \nu-1} w\right)^{\prime}\right)^{\prime}=(r / \gamma)\left(u^{q}-u\right)$ and $u^{q}-u<1-q$ for $u>0$, we have

$$
\left(r^{1-2 \nu}\left(r^{2 \nu-1} w\right)^{\prime}\right)^{\prime}(r)<\frac{(1-q) r}{\gamma}, \quad r>0 .
$$

Integrating (3.48) from $r_{0}$ to any point $r>r_{0}$ and using the estimate (3.37) at $r_{0}$, we find

$$
r^{1-2 \nu}\left(r^{2 \nu-1} w\right)^{\prime}(r)<-\frac{1}{2}|h(a)|+\frac{(1-q) r^{2}}{2 \gamma}, \quad r>r_{0}
$$

for all $\gamma \geq \gamma_{1}$. Because $\gamma \delta^{2}=\gamma-\gamma^{q}>\gamma^{1-q}-1>(1-q) \log \gamma$, it follows that

$$
r^{1-2 \nu}\left(r^{2 \nu-1} w\right)^{\prime}(r)<-\frac{1}{2}|h(a)|+\frac{r^{2} \delta^{2}}{2 \log \gamma}, \quad r>r_{0}
$$

for all $\gamma \geq \gamma_{1}$.

Now, we restrict $r$ to a compact interval $\left[r_{0}, r_{2}\right]$ where

$$
r_{2}=b / \delta
$$

and $b>a$ is a suitably chosen constant. Defining the constant $\gamma_{2}$ by

$$
\gamma_{2}=\min \left\{\gamma_{1}, \mathrm{e}^{2 b^{2} /|h(a)|}\right\},
$$

we then have

$$
\frac{r^{2} \delta^{2}}{2 \log \gamma} \leq \frac{1}{4}|h(a)|, \quad r_{0} \leq r \leq r_{2},
$$

for all $\gamma \geq \gamma_{2}$, so (3.50) reduces to

$$
r^{1-2 \nu}\left(r^{2 \nu-1} w\right)^{\prime}(r)<-\frac{1}{4}|h(a)|, \quad r_{0} \leq r \leq r_{2}
$$

for all $\gamma \geq \gamma_{2}$. Hence,

$$
w\left(r_{2}\right)<\left[(a / b)^{2 \nu} \frac{w\left(r_{0}\right)}{r_{0}}-\left(1-(a / b)^{2 \nu}\right) \frac{|h(a)|}{8 \nu}\right] r_{2} .
$$

Using (3.38) to estimate $w\left(r_{0}\right) / r_{0}$ and writing the inequality in terms of $u$, we thus find that

$$
u\left(r_{2}, \gamma\right)<(a / b)^{2 \nu}\left(1+\frac{4 \nu}{|h(a)|}\right)^{1 /(2 \nu)}-\gamma\left(1-(a / b)^{2 \nu}\right) \frac{|h(a)|}{8 \nu}
$$

for all $\gamma \geq \gamma_{2}$.

But now we have a contradiction, as the expression on the right-hand side of this inequality certainly becomes negative for sufficiently large values of $\gamma$. We conclude, therefore, that $u(\cdot, \gamma)$ reaches the value 0 at some finite point $r_{1}$, as claimed.

3.6. Completion of the Proof. According to Lemma $7, u(\cdot, \gamma)$ ceases to be positive at a finite point $r_{1}$ for all $\gamma \geq \gamma_{2}$ where $\gamma_{2}$ is a constant that does not depend on $q$. Obviously, $r_{1}$ depends on the value of $\gamma$; in fact, it decreases as $\gamma$ increases. Let

$$
\Gamma=\inf \left\{\gamma>1: r_{1}<\infty\right\} \text {. }
$$

If $\gamma=u(0)=\Gamma$, then $u(\cdot, \gamma)$ reaches the $r$-axis with a horizontal slope, so $u(\cdot, \Gamma)$ defines the unique solution $u$ of the free-boundary problem (1.1), (1.2) where

$$
R=r_{1}(\Gamma) \text {. }
$$

Obviously, $\Gamma$ depends on $q$. However, it follows from Lemma 7 that $1<\Gamma \leq \gamma_{2}$, so $u(0)$ is bounded as $q \rightarrow 1(q<1)$. 
It remains to investigate the behavior of $R$ as $q \rightarrow 1(q<1)$. Because $\Gamma$ is bounded, $\lim _{q \rightarrow 1} \Gamma^{1-q}=1$. Then it follows from (3.12) that $\lim _{q \rightarrow 1} \delta=0$ and, therefore, by Lemma $3, \lim _{q \rightarrow 1} R=\infty$. Thus, the proof of the theorem is complete.

3.7. Special Case: $N=3$. In the special case $N=3\left(\nu=\frac{1}{2}\right)$, it actually is possible to find a lower bound for $R$ that shows that $R$ grows beyond bounds as $q \rightarrow 1$.

A simple energy argument gives the inequality

$$
0=E(R)<E(0)=\frac{\Gamma^{2}}{2}-\frac{\Gamma^{1+q}}{1+q}
$$

cf. (3.7). Hence,

$$
\Gamma^{1-q}>\frac{2}{1+q}
$$

Next, we use an energy argument for (3.9). If $\nu=\frac{1}{2}$, this equation reduces to

$$
w^{\prime \prime}+w-\Gamma^{-(1-q)} r^{1-q} w^{q}=0 .
$$

Hence,

$$
\left(w^{\prime 2}+w^{2}-\frac{2}{1+q} \Gamma^{-(1-q)} r^{1-q} w^{1+q}\right)^{\prime}=-2 \frac{1-q}{1+q} \Gamma^{-(1-q)} r^{-q} w^{1+q}
$$

Upon integration over $(0, R)$, the left-hand side yields -1 ; on the right-hand side, we use the inequality $w(r)<r$ to obtain the estimate

$$
\int_{0}^{R} r^{-q} w^{1+q} d r<\frac{1}{2} R^{2}
$$

Thus, using (3.60), we find that

$$
R>\sqrt{\frac{2}{1-q}} .
$$

Acknowledgment. This work was supported by the Mathematical, Information, and Computational Sciences Division subprogram of the Office of Computational and Technology Research, U.S. Department of Energy, under Contract W-31-109-Eng-38.

\section{References}

1. H. G. Kaper and M. K. Kwong, Free boundary problems for Emden-Fowler equations, Differential and Integral Equations 3 (1990), 353-362.

2. G. Miller, V. Faber, and A. B. White, Jr., Finding plasma equilibria with magnetic islands, J. Comp. Phys. 79 (1988), 417-435.

3. G. N. Watson, A Treatise on the Theory of Bessel Functions, 2nd ed. Cambridge University Press, 1966.

Department of Mathematics, University of Toronto, Toronto, M5S 1A1, Ontario, Canada

Mathematics and Computer Science Division, Argonne National Laboratony, Argonne, IL 60439 